\title{
Spectral and Photometric Evolution of V725 Tau (1996-1999): Towards a Shell Phase?
}

\author{
A. Piccioni ${ }^{1}$, C. Bartolini ${ }^{1}$, S. Bernabei ${ }^{1}$, S. Galleti ${ }^{1}$, F. Giovannelli ${ }^{2}$, \\ A. Guarnieri ${ }^{1}$, L. Sabau-Graziati ${ }^{3}$, G. Valentini ${ }^{4}$ and M. Villada ${ }^{5}$ \\ ${ }^{1}$ Dipartimento di Astronomia, Universitá di Bologna, Italy \\ ${ }^{2}$ Istituto di Astrofisica Spaziale, Frascati, Italy \\ ${ }^{3}$ I.N.T.A., Madrid, Spain \\ ${ }^{4}$ Osservatorio di Collurania, Teramo, Italy \\ ${ }^{5}$ C.A.S.L.E.O., Argentina
}

\begin{abstract}
.
HDE $245770=$ V725 Tau, the optical counterpart of A0535+26, presented in the last three years a period of uncommon optical faintness and of exceptionally low spectral activity. Photometric data obtained in 1997 and 1998 show a fading in all color, followed in 1999 by a nearly constant phase in which the star attained the lowest level of the last 23 years. In 1998 the emission lines became much fainter than in 1997, changing slowly from emission to absorption. In November 1998 only $\mathrm{H} \alpha$ was still showing a weak emission with an absorption core; this behaviour suggests the occurrence of a shell phase.
\end{abstract}

\section{Introduction}

V725 Tau $=$ HDE $245770=\mathrm{A} 0535+26$ is a well known Be-X-ray system which shows a behaviour characterized by a large variety of phenomena. After the discovery of A0535+26 as an X-ray transient by ARIEL 5, our group confirmed the proposed identification of HDE 245770 as its optical counterpart (Bartolini et al, 1978), classified the spectrum (Giangrande et al, 1980), observed some optical flares precursors of the X-ray activity (Bartolini et al., 1979; Guarnieri et al. 1982; Giovannelli et al, 1990), found optical evidence of orbital modulation (exploiting an unexpected phase of quiescence of the Be star activity; Guarnieri et al. 1985) and observed an episode of unusual spectral activity probably connected to the transfer of a blob of matter from the Be star to the collapsed companion (Burger et al. 1996). In order to give a full description of this source, the behaviour in the other spectral ranges should be added to this rich optical phenomenology: the X-ray pulsar emission with a period of $104 \mathrm{~s}$, the different appearance of X-ray outbursts, the presence of the cyclotron resonant line at $110 \mathrm{keV}$ and of magnetic field strength $\geq 10^{13}$ Gauss (Maisak et al. 1997), the detection of QPOs in outburst (Finger et al. 1996), a stable (in outburst and in quiescence) terminal velocity of the stellar wind of $\sim 600 \mathrm{~km} / \mathrm{s}$, deduced by 


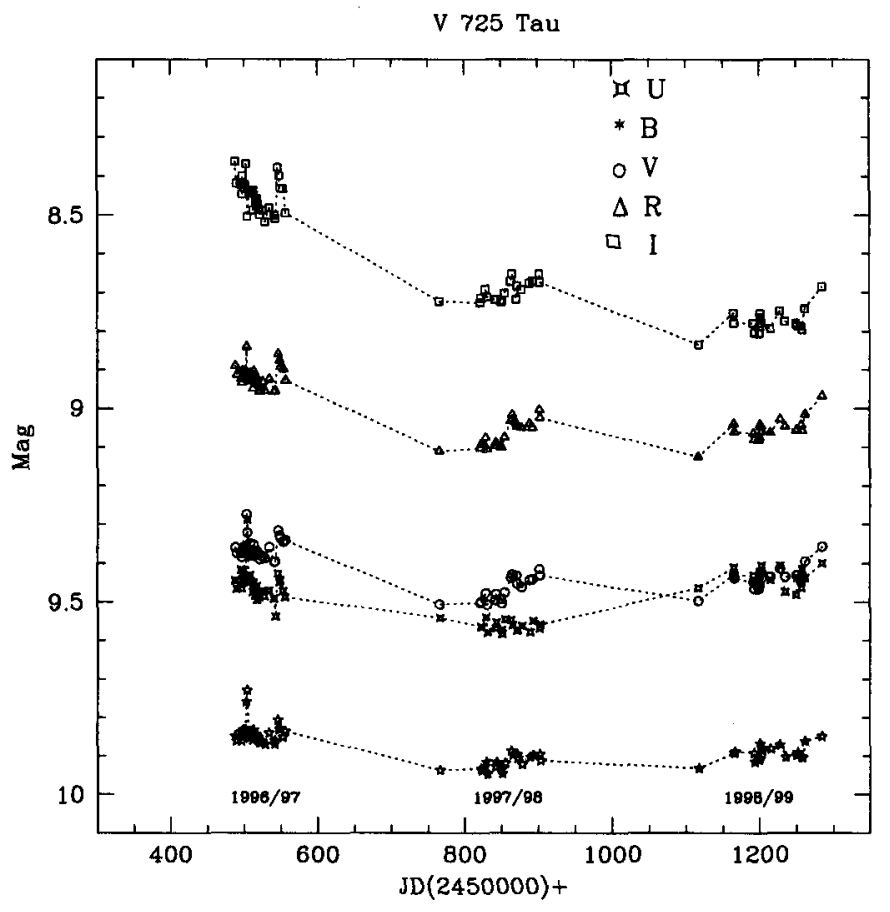

Figure 1. UBVRI photometrical light curve during the period 19961999

the analysis of resonance lines in the UV band (Giovannelli et al. 1982) and the strong IR excess due to contribution of thermal free-free radiation in the ionized envelope of the $\mathrm{Be}$ component (de Loore et al. 1984). The resulting picture (Giovannelli \& Sabau-Graziati, 1992) describes a binary system composed by a O9.7IIIe primary star and a neutron star in an orbit eccentric enough to start the mass transfer and the type I outburst near the periastron only.

\section{Photometry}

The photometric observations we present here were performed with the UBVRI sequential photon counting photometer, mounted on the Cassegrain focus of the $62 \mathrm{~cm}$ ZEISS telescope of the Bologna University, at Loiano. They refer to 69 photometric nights: 31 in 1996-97, 19 in 1997-98 and 19 in 1998-99. Fig. 1 shows the light curves in each color band. A general fading is present in the period between late 1996-early 1998; the trend of the color indices during the same period seems to indicate a decrease of the infrared excesses. In the period from early 1998 to early 1999 the luminosity was constant or showed a slight tendency to increase in the B and V bands. Two short enhancements $(\sim 0.1$ mag of amplitude) were observed on February 23-24 and April 7, 1997; the steep rise is followed by a slower fading, as in the case of the optical precursors of the 

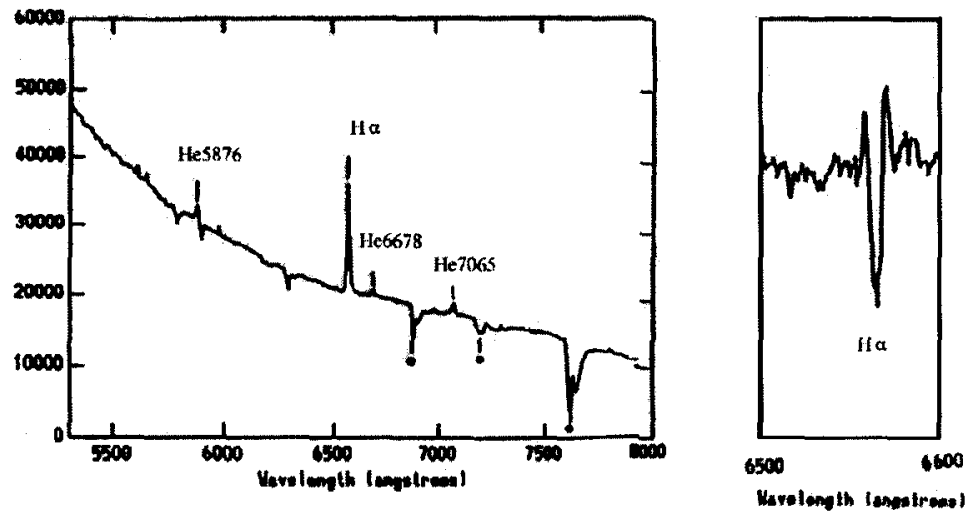

Figure 2. A typical spectrum of V725 Tau (1997.02.19, left) showing $\mathrm{H} \alpha$ and $\mathrm{He} \mathrm{I}$ emission. The $\mathrm{H} \alpha$ absorption core during the shell phase (1998.11.10, right). Intensities are in arbitrary scale.

X-ray activity already observed, but in this case without a corresponding X-ray activity; the red color of these short flares favours their interpretation as episodes of ejection of matter from the Be star, which feeds its equatorial disk. However the clear evidence of a long term decrease of the U-B and R-I indices during the season 1996-1999 indicates a tendency to the depletion of circumstellar disk. With these new data we updated the longterm light curve (Piccioni et al, 1998 and references therein). V725 Tau is presently at the lowest luminosity level in its history. The luminosity decay of the last years looks quite similar to the decline observed in Pleione in the seventies during the transition from the $\mathrm{Be}$ phase to the shell phase (Sharov \& Lyuty, 1976).

\section{Spectroscopy}

The spectra have been obtained with the BFOSC spectrograph, mounted on the $152 \mathrm{~cm} \mathrm{G.D.} \mathrm{Cassini} \mathrm{telescope} \mathrm{at} \mathrm{Loiano;} \mathrm{the} \mathrm{low} \mathrm{resolution} \mathrm{does} \mathrm{not} \mathrm{allow}$ a deep analysis of the line profiles; only red spectra were taken, owing to the low sensitivity in the blue of the LORAL CCD detector. In spite of the low quality of the spectra and of the lack of information on the higher members of the Balmer line series, a dramatic change in the envelope of the Be star is shown by the progressive fading of $\mathrm{He}$ I 5876, 6678 and 7805 lines, which from 1997 to 1998 changed from emission to absorption and by the evolution of $\mathrm{H} \alpha$ (Fig. 2), whose equivalent widths are reported in Fig. 3. A medium dispersion spectrum taken on November 1998 on the CASLEO Observatory (Fig. 2 right) shows that $\mathrm{H} \alpha$ was developing a deep absorption core between two wings in emission, as is typical of the shell phase in Be stars (whose Pleione and 59 Cyg are well known examples; Underhill \& Doazan, 1982). Also in the spectra of 1999 a trace of the central absorption typical of this phase is present, somewhat hidden by the low resolution. 


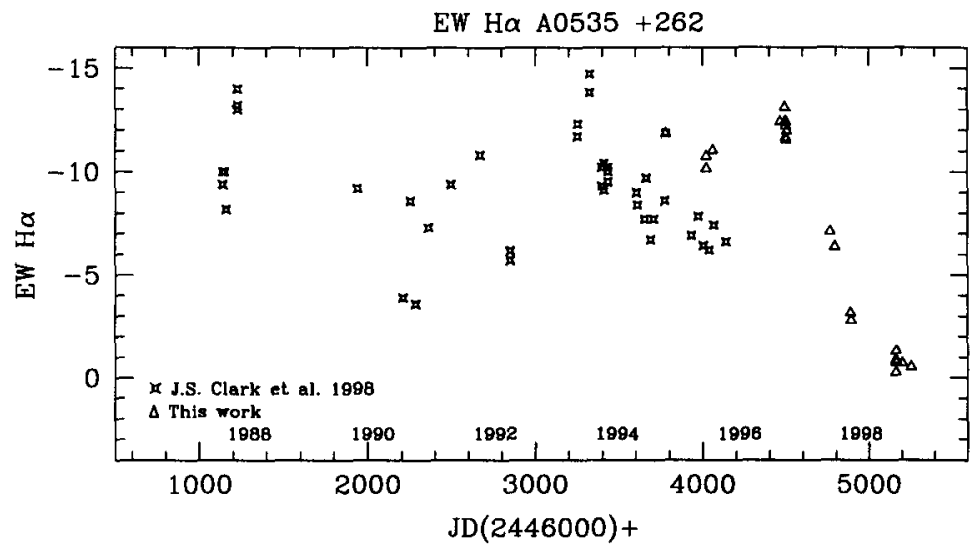

Figure 3. $\mathrm{H} \alpha$ equivalent width evolution during the last decade

\section{Conclusions}

In the present phase, V725 Tau $=A 0535+26$ is probably at its faintest luminosity level of this century. Correspondingly, the Balmer and He I emission line system completely disappeared or evolved in absorption. In the case of $\mathrm{H} \alpha$ it is worthwhile to note that it was the first time that this happened since the discovery of HDE 245770 as the optical counterpart of A0535 +26. The strong photometric fading, the color indices evolution and the spectral behaviour are indicative of the fact that V725 Tau is probably running to a shell phase.

\section{References}

Bartolini, C. et al. 1978, IAU Circ. 3167.

Bartolini, C. et al. 1979, IAU Circ. 3367.

Burger, M. et al. 1996, Mem S.A.It. 67, 365.

Clark, J.S. et al. 1998, MNRAS 294, 165.

De Loore, C. et al. 1984, A\&A 141, 279.

Finger, M.H. et al. 1996, ApJ 459, 288.

Giangrande, A. et al. 1980, A\&AS 40, 289.

Giovannelli, et al. 1982, ESA SP 179, 233.

Giovannelli, F. et al. 1990, Ap\&SS 169, 139.

Giovannelli, F., Sabau Graziati, L. 1992, Space Sci.Rev. 59, 1.

Guarnieri, A. et al. 1982, IAU Symp. 98, 69.

Guarnieri, et al., 1985 Frascati Workshop 1984, 310.

Maisak, M. et al. 1997, A\&A 325, 212.

Piccioni, A. et al 1998, Vulcano Workshop 1998, in press.

Sharov, A.S., Lyuty, V.M. 1976, Proc IAU Symp. 70, 105.

Underhill, A., Doazan, V. 1982, NASA SP-456 11, 285. 